\title{
Argos induces programmed cell death in the developing Drosophila eye by inhibition of the Ras pathway
}

\author{
Kazunobu Sawamoto ${ }^{1}$, Akiko Taguchi ${ }^{1}$, Yuki Hirota ${ }^{1}$, \\ Chiharu Yamada ${ }^{1}$, Ming-hao $\mathrm{Jin}^{1}$ and Hideyuki Okano ${ }^{1,2}$ Medical School, and CREST, Japan Science and Technology Corporation (JST), 2-2 Yamadaoka, Suita, Osaka 565, Japan
2 corresponding author: Hideyuki Okano, Department of Neuroanatomy, Biomedical Research Center, Osaka University Medical School, 2-2 Yamadaoka, Suita, Osaka 565, Japan. tel: 81-6-879-3581; fax 81-6-879-3589; \\ 1 Department of Neuroanatomy, Biomedical Research Center, Osaka University \\ email: okano@nana.med.osaka-u.ac.jp
}

Received 30.8.97; revised 7.10.97; accepted 24.10.97

Edited by S. Nagata

\begin{abstract}
We studied the role of Ras signaling in the regulation of cell death during Drosophila eye development. Overexpression of Argos, a diffusible inhibitor of the EGF receptor and Ras signaling, caused excessive cell death in developing eyes at pupal stages. The Argos-induced cell death was suppressed by coexpression of the anti-apoptotic genes p35, diap1, or diap2 in the eye as well as by the $\mathrm{Df}(3 \mathrm{~L}) \mathrm{H} 99$ chromosomal deletion that lacks three apoptosis-inducing genes, reaper, head involution defective (hid) and grim. Transient misexpression of the activated Ras1 protein (Ras $1^{\mathrm{V} 12}$ ) later in pupal development suppressed the Argos-induced cell death. Thus, Argos-induced cell death seemed to have resulted from the suppression of the anti-apoptotic function of Ras. Conversely, cell death induced by overexpression of Hid was suppressed by gain-of-function mutations of the genes coding for MEK and ERK. These results support the idea that Ras signaling functions in two distinct processes during eye development, first triggering the recruitment of cells and later negatively regulating cell death.
\end{abstract}

Keywords: Argos; apoptosis; compound eye; Drosophila; programmed cell death; Ras

Abbreviations: PCD, programmed cell death; rl, rolled; Dsor1, Downstream suppressor of raf 1; PBS, phosphate buffered saline; IAP, Inhibitor of apoptosis protein; DIAP1, Drosophila IAP1; DIAP2, Drosophila IAP2; hid, head involution defective; $r p r$, reaper, DER, Drosophila EGF receptor, ICE, interleukin-1 $\beta$ converting enzyme

\section{Introduction}

Programmed cell death (PCD), or apoptosis, is a form of cell death in which supernumerary or harmful cells are removed from an organism. Many lines of evidence indicate that PCD is an active, gene-directed process, the underlying mechanisms of which are highly conserved throughout animal evolution (Steller, 1995; White and Steller, 1995). The genetic cascade underlying PCD has been most intensively characterized in the nematode, Caenorhabditis elegans (reviewed by Ellis et al, 1991; Hengartner and Horvitz, 1994). In particular, three nematode genes, ced-9, ced-4, and ced-3 have been identified as PCD regulators. Of these, ced-3 and ced-4 are required for all PCD onset (Ellis and Horvitz, 1986), whereas ced-9, in turn, suppresses the PCD induced by ced-3 and ced4 (Hengartner et al, 1992). The ced-9 gene is homologous to the human anti-apoptotic gene bcl-2 (Tsujimoto et al, 1985; Hengartner and Horvitz, 1994). In addition, a mammalian gene Apaf- 1 that shows significant homology with ced-4 has been identified recently (Zou et al, 1997). The ced-3 gene is homologous to a family of cysteine proteases, called caspases, which includes the interleukin-1 $\beta$-converting enzyme (ICE) (Yuan et al, 1993; Miura et al, 1993). It has been shown that the inhibition of caspase activity by the baculovirus p35 protein can prevent PCD in nematodes (Sugimoto et al, 1994), insects (Hay et al, 1994) and mammals (Rabizadeh et al, 1993). Thus, components of the PCD pathway have been highly conserved throughout evolution. Despite good progress in identifying cell death genes, we do not understand much about the molecular mechanisms of PCD at present. The fruit fly, Drosophila melanogaster, provides a powerful tool for investigating the mechanisms of PCD using a number of sophisticated genetic strategies.

In Drosophila, three genes, reaper (rpr) (White et al, 1994), head involution defective (hid) (Grether et al, 1995), and grim (Chen et al, 1996) have been identified as playing a central role in the initiation of PCD. Genetic studies have revealed that these three genes are likely to function independently to induce PCD via a caspase pathway (White et al, 1996; Grether et al, 1995; Chen et al, 1996). Among these genes, rpr has been best characterized. The transcription of rpr is activated by stimuli that cause PCD, such as X-ray irradiation and aberrant development (Nordstorm et al, 1996). Thus, the induction of rpr expression (and also of grim and hid, possibly) and the subsequent activation of the caspase family are likely to represent a 'central pathway' for PCD in Drosophila. PCD in Drosophila is known to be controlled by a number of distinct signals, including extra- and intracellular signals, blockage of cell differentiation, and X-ray induced damage (reviewed by McCall and Steller, 1997). However, the signaling machineries by which the various stimuli activate the central pathway of PCD remain largely unknown.

The Drosophila compound eye is a useful system for studying the mechanisms of PCD as well as cell-fate decisions. The compound eye is composed of about 750 repeating units called ommatidia. Each ommatidium contains the same number of cells, including eight 
photoreceptor cells (R1-8), four cone cells (lens-secreting cells) and two primary pigment cells. There are also six secondary and three tertiary pigment cells, shared by neighboring ommatidia. Since each ommatidium is composed of exactly the same complement of cells, it is tempting to speculate that there are strict regulatory mechanisms controlling the precise number of each cell type within an ommatidium. An increasing number of such regulatory cues have been shown to be involved in this process: e.g., an inductive event mediated by receptor tyrosine kinases (reviewed by Freeman, 1997) and lateral inhibition accomplished through several mechanisms (reviewed by Sawamoto and Okano, 1996). PCD also plays an important role in regulating the number of cells within each ommatidium.

It is known that massive PCD occurs in the pupal eye, resulting in the elimination of two to three cells per ommatidium (Wolff and Ready, 1991). This event is known to be required as a final step in pattern formation to eliminate supernumerary cells associated with each cluster and to establish the highly ordered lattices of secondary and tertiary pigment cells (Cagan and Ready, 1989; Bonini, 1997). It is generally known that PCD is induced in the Drosophila compound eye when normal development, including cell recruitment, is impaired by mutations or events (reviewed by Bonini, 1997). However, much remains to be elucidated about the common regulatory mechanisms required for both cell recruitment and $P C D$ in the developing eye disc. The Ras signaling pathway has been shown to be required for triggering the differentiation of all the cell types in the eye (reviewed by Freeman, 1997). Although Ras has also been implicated in PCD in other systems (reviewed by Pritchard and McMahon, 1997), the role of the Ras pathway in the regulation of PCD during Drosophila development is still unclear. In this regard, Freeman (1994) has reported a noteworthy observation that overexpression of Argos, a secreted protein with an EGFlike domain that acts as an inhibitor for the Drosophila EGF receptor (DER) and the Ras pathway (Okano et al, 1992; Freeman et al, 1992; Kretzschmar et al, 1992; Schweitzer et al, 1995a; Sawamoto et al, 1996), causes excessive cell death. This result suggests involvement of Ras signaling in PCD in the developing eye. In the present study, we have characterized the cell death caused by the overexpression of Argos in detail and found a novel function of Ras signaling that prevents PCD in the eyes.

\section{Results \\ Overexpression of argos driven by an eye-specific promoter causes cell death during pupal development}

To target argos overexpression specifically to the eye, we used the pGMR vector to generate transgenic flies in which argos expression was under the control of tandemly-arrayed regulatory elements of the eye-specific transcription factor Glass (Hay et al, 1994). The phenotype of the transgenic flies carrying one copy of the GMR-argos transgene was similar to that of HS-argos (Sawamoto et al, 1994; Freeman, 1994;
Brunner et al, 1994), i.e., a decrease in the numbers of photoreceptor, cone, and pigment cells was observed (see Figure 3). The phenotype appeared to be sensitive to the dosage of the transgene, since flies carrying more copies of the GMR-argos transgene showed much stronger phenotypes (data not shown). In this study, we always used flies with one copy of the GMR-argos transgene. To determine if overexpressed Argos caused cell death, we stained live discs with acridine orange. In eye discs from wild-type thirdinstar larvae, dying cells stained with acridine orange were seen both anterior and posterior to the morphogenetic furrow (Figure 1A), as previously described (Wolff and Ready, 1991). The pattern of cell death in the GMR-argos larval discs (Figure 1B) was indistinguishable from wild-type (Figure 1A). We also examined photoreceptor differentiation by labeling larval eye discs with anti-Elav antibody, a nuclear marker for all Drosophila neurons. No obvious defects were discerned in the GMR-argos eyes (data not shown). Approximately $32-35 \mathrm{~h}$ after puparium formation (APF), massive PCD occur in the wild-type eyes to eliminate excess cells (Wolff and Ready, 1991; Figure $1 C)$. In the GMR-argos eyes, the number of dead cells was
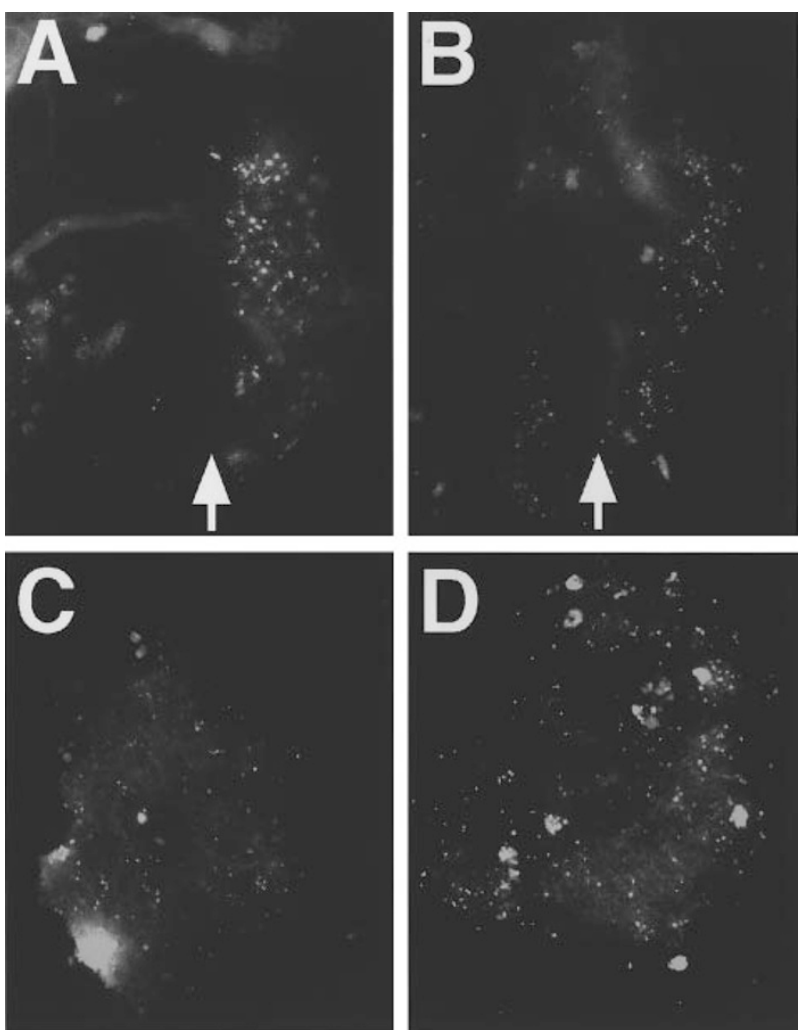

Figure 1 Overexpressed Argos induces excessive cell death in the pupal retina (A) Wild-type third instar eye-antennal disc stained with acridine orange. A small number of dying cells are observed within the eye disc. Anterior is to the left. (B) Third instar eye-antennal disc from GMR-argos/+larva. The level of cell death is indistinguishable from wild-type. Anterior is to the left. (C) Wildtype retina at $35 \mathrm{~h}$ APF. Excess pigment cells stained with acridine orange are eliminated by apoptosis at this stage. (D) Retina from GMR-argos/+pupa at $35 \mathrm{~h}$ APF. The number of dying cells stained with acridine orange is significantly increased over wild-type. The arrows in (A) and (B) indicate the position of the morphogenetic furrow 

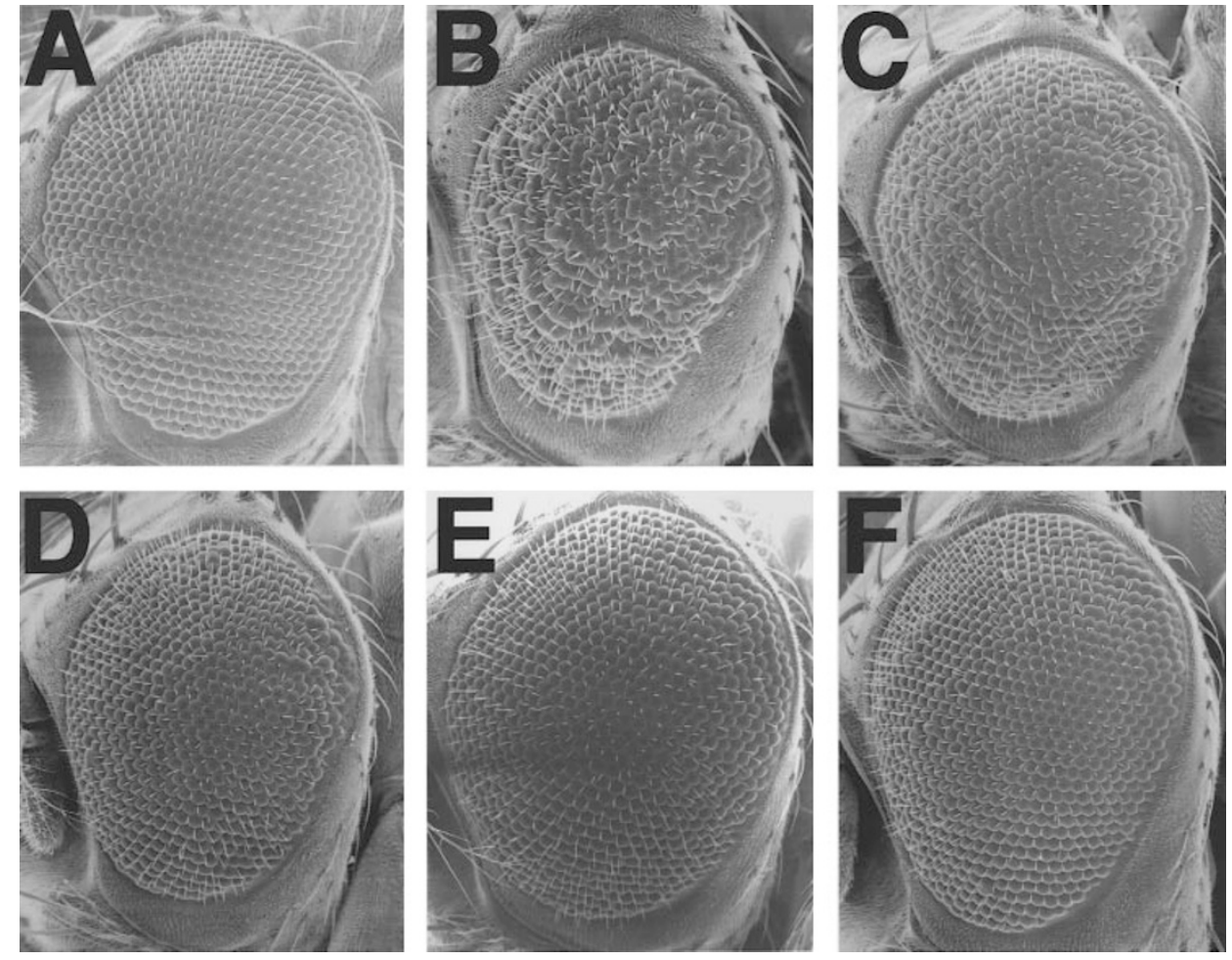

Figure 2 The phenotype of the GMR-argos eye is suppressed by co-expression of anti-apoptotic genes and in the H99 mutation background. (A) Wild-type compound eye, composed of a regular array of about 750 ommatidia. (B) GMR-argos. Note the slight reduction in size and the rough appearance. The phenotype of GMR-argos is significantly suppressed by co-expression of the anti-apoptotic genes p35 (C), diap1 (D), and diap2 (E). The H99 deletion also acts as a dominant suppresser of GMR-argos (F)

considerably increased in the pupal retina (Figure 1D), as observed previously for HS-argos eyes (Freeman, 1994). Thus, one copy of the GMR-argos transgene is unlikely perturb the neuronal differentiation and cell death in larval discs, but induces excessive cell death during pupal development.

\section{Cell death in the GMR-argos eyes is mediated by caspases and apoptotic activators encoded in the 75C1-2 region}

To analyze the mechanisms underlying the excessive cell death in the pupal eyes caused by Argos overexpression, we examined the effects of coexpressing anti-apoptotic factors that act as inhibitors for caspases. Expression of baculovirus p35, which acts as an inhibitor of caspases (Bump et al, 1995), is known to prevent PCD in Drosophila (Hay et al, 1994). Expression of either Diap1 or Diap2, Drosophila homologues of the baculovirus inhibitor of apoptosis proteins (IAPs), also prevents PCD in the eye (Hay et al, 1995). GMRargos flies were crossed to the files in which p35, diap1, or diap2 were expressed specifically in the eyes using the pGMR vector. All of the GMR-p35 (Hay et al, 1994), GMR-diap1 (Hay et al, 1995), and GMR-diap2 (Hay et al, 1995) flies have a mild rough-eye phenotype due to the blockage of normally occurring cell death. GMR-argos eyes have a severely rough phenotype and a slightly smaller size (Figure $2 \mathrm{~B}$ ) compared with wild-type (Figure 2A). The GMR-argos phenotype was significantly suppressed by the coexpression of any of the anti-apoptotic genes, p35 (Figure 2C), diap1 (Figure 2D), and diap2 (Figure 2E), implying that the overexpression of Argos caused PCD through a common apoptotic pathway that included the activation of caspases.

It is known that the 75C1-2 region of the Drosophila genome is essential for all PCD that normally occur during embryogenesis (White et al, 1994). The Df(3L)H99 deletion mutation lacks at least three genes coding for the apoptotic activators, rpr (White et al, 1994), hid (Grether et al, 1995), and grim (Chen et al, 1996) in this $75 \mathrm{C} 1-2$ region. To analyze the effects of halving the dosage of genes in this region on the PCD caused by overexpressed Argos in the eyes, we crossed the GMR-argos flies to the $D f(3 L) H 99$ mutants. As shown in Figure $2 \mathrm{~F}$, the $\mathrm{Df}(3 \mathrm{~L}) \mathrm{H} 99$ mutation dominantly suppressed the eye phenotype of the GMR-argos. Therefore, gene(s) at 75C1-2 such as rpr, hid, or grim are likely to play essential roles in PCD in the GMR-argos eyes.

To determine the cell types that were eliminated by PCD from the GMR-argos eyes, sections of adult heads and pupal retinae stained with cobalt sulfide were examined (Figure 3). In wild-type flies, each ommatidium contains eight photoreceptor cells, seven of which can be observed in one tangential section (Figure $3 \mathrm{~A}$ ). Secondary and tertiary pigment cells form highly ordered pigmented lattices surrounding each ommatidium (Figure $3 A$ ). The 

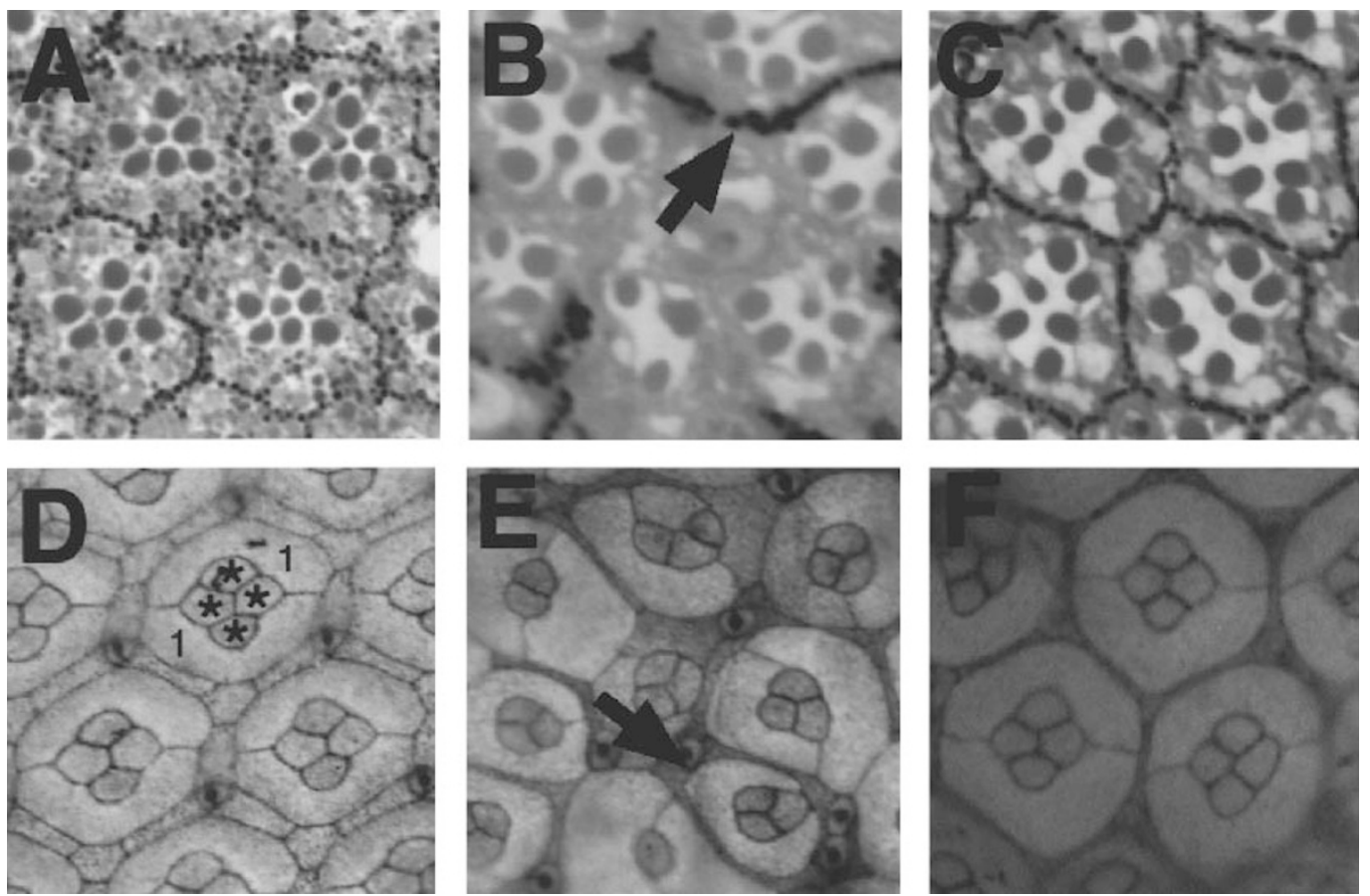

Figure 3 Expression of diap1 restores all the cell types in the GMR-argos eyes. (A-C) Sections of adult eyes of wild-type (A), GMR-argos/+(B) and GMR-diap1/ +; GMR-argos/+(C) flies. (A) In wild-type, each ommatidium contains eight photoreceptor cells, seven of which can be observed in one tangential section. Secondary and tertiary pigment cells form pigmented lattices surrounding each ommatidium. (B) The GMR-argos/+ eye shows a reduced number of photoreceptor cells and loss of pigmented lattice. The residual pigmented lattices are marked by an arrow. (C) Co-expression of diap1 restores the photoreceptor cells and pigmented lattice in the GMR-argos eye. (D-F) Cobalt sulfide staining of the wild-type (D), GMR-argos/+ (E), and GMR-diap1/+; GMR-argos/+ (F) flies. (D) In the wild-type eye, the apical surface of each ommatidium displays the outline of four cone cells (indicated by asterisks) and two primary pigment cells (indicated by 1 ). (E) Overexpression of argos causes a reduction in the number of cone and primary pigment cells. The arrow indicates an ommatidium that contains three cone cells and one primary pigment cell. (F) Normal numbers of both cone and primary pigment cells are seen in the GMR-diap1/+; GMR-argos/+ommatidia

GMR-argos eye showed a reduced number of photoreceptor cells and loss of pigmented lattices (Figure 3B). This phenotype was almost completely suppressed by the coexpression of diap1 (Figure 3C). The apical surface of each ommatidium on the wild-type retinae at $40 \mathrm{~h}$ APF displays the outline of four cone cells and two primary pigment cells (Figure 3D). Overexpression of argos caused a reduction in the number of cone and primary pigment cells (Figure $3 \mathrm{E}$ ). In contrast, in the GMR-argos/GMR-diap1 eyes, we observed the normal number of cone and primary pigment cells in most of the ommatidia (Figure 3F). Coexpression of either p35 or diap2, or the $D f(3 L) H 99$ mutation, also showed similar suppressive effects on the GMR-argos phenotype (data not shown). These results indicate that loss of all the cell types in the GMR-argos eyes is caused by the enhanced PCD, mediated - at least in part - through the actions of caspases and the cell-death activators encoded by a gene or genes in the $75 \mathrm{C} 1-2$ region.

\section{Ras activation later in pupal development prevents cell death in GMR-argos eyes}

It is known that PCD is enhanced when cell recruitment is impaired in the developing Drosophila eye disc (reviewed by Bonini, 1997). Thus, it is possible that the increased PCD in the GMR-argos eyes is the result of impaired cell recruitment due to the inhibition by overexpressed Argos of EGF receptor signaling and subsequent Ras signaling, since EGF receptor signaling is known to be required to trigger the differentiation of all the cell types in the eye (Freeman, 1996). Alternatively, the Ras signaling activity, which is suppressed by the overexpressed Argos, could regulate PCD without affecting the recruitment of retinal cells. To test this possibility, we examined the effect of transiently expressing the constitutively activated Ras 1 protein (Ras $1^{\mathrm{V} 12}$ ), a downstream effector of the EGF receptor, under the control of the heatinducible $h s p 70$ promoter. The wild-type ommatidium is composed of four cone cells, two primary pigment cells, six secondary pigment cells, and three tertiary pigment cells (Figure 4A). Transient expression of Ras $1^{\mathrm{V} 12}$ by heat shock during early pupal development $(0-5.5 \mathrm{~h}$ APF) caused an increase in cone cell-like cells (Figure 4B). Heat shocks during late pupal development (20-40 h APF), however, had no effect on cone cell formation, but caused an increase in the number of secondary and tertiary pigment cells (Figure 4C). The time at which over-recruitment of each cell type is induced by expression of the activated Ras 1 protein (summarized in Figure 4D) corresponds well with the start of its differentiation (Cagan and Ready, 1989) and the time at which each cell type is sensitive to the inhibition of DER function (Freeman, 1996).

We utilized the cell-type specific effect of transient Ras $1^{\mathrm{V} 12}$ expression to examine the function of Ras 1 in 

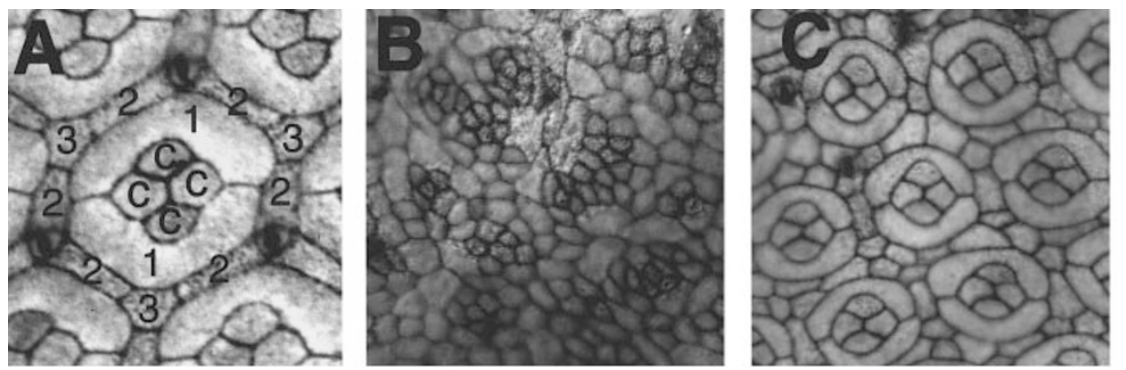

D

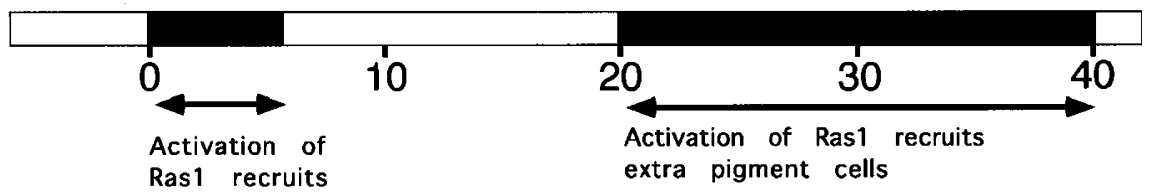

Ras1 recruits

extra cone cells
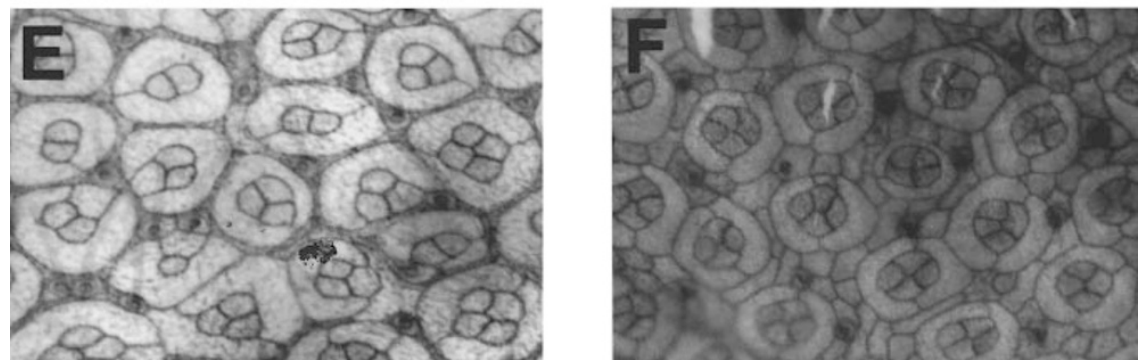

Figure 4 Effects of transient Ras activation on eye development at the pupal stage. Cobalt sulfide staining of retinae at $40 \mathrm{~h} \mathrm{APF}$. (A) In the wild-type ommatidium, four cone cells $(c)$, two primary pigment cells (1), six secondary pigment cells (2), and three tertiary pigment cells (3) are visible. This pattern was not altered by heat-shock at any time during pupal development. (B-C) Expression of Ras $1^{\mathrm{V} 12}$ was induced at $0-6 \mathrm{~h}(\mathrm{~B})$ or $20-40 \mathrm{~h}(\mathbf{C})$ with heat shocks and stained with cobalt sulfide at $40 \mathrm{~h}$ APF. (B) Heat shocks at early pupal stage cause recruitment of extra cone-cell like cells. (C) Heat shocks at $20-40 \mathrm{~h}$ APF cause recruitment of extra pigment cells but do not affect the cone cell number. (D) Summary of the effects of Ras1 activation at different times in eye development of wild-type flies. The times

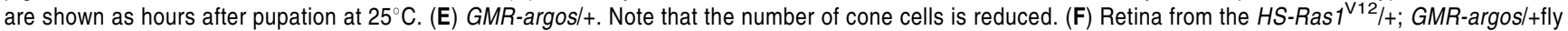
heat shocked at 20-40 h APF. Most of the ommatidia contain normal numbers of cone cells

regulating PCD in the GMR-argos eyes. Here, we focused on cone cell death, since we could not distinguish the effect of Ras $1^{\mathrm{V} 12}$ on the differentiation of pigment cells from its effect on the regulation of PCD (see Discussion). The HS-

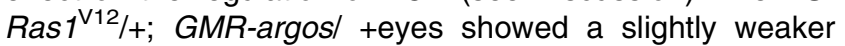
phenotype than GMR-argos even without heat shock (data not shown), suggesting that $\operatorname{Ras} 1^{\mathrm{V} 12}$ expression is constitutively induced at a low level at $25^{\circ} \mathrm{C}$. Flies carrying both the GMR-argos and HS-Ras $1^{\mathrm{V} 12}$ transgenes were heat-shocked at the late pupal stage (20-40 h APF) to exclude the effects of HS-Ras $1^{\mathrm{V} 12}$ on the recruitment of cone cells. The transient expression of $\operatorname{Ras}^{\mathrm{V} 12}$ at $20-40 \mathrm{~h}$ APF caused a variable but significant reduction in the loss of cone cells in the GMR-argos eyes. Heat shocks at 20$40 \mathrm{~h}$ APF did not cause any visible effect on eye development in the flies without the HS-Ras $1^{\mathrm{V} 12}$ transgene: about half of the ommatidia contained reduced numbers of cone cells in the GMR-argos flies (Figure 4E). Heat shocks of the HS-Ras $1^{\mathrm{V} 12} /+$; GMR-argos/+flies at the same stage, however, significantly increased the population of the ommatidia that contained four cone cells (Figure 4F). Therefore, the cone cell death in the GMR-argos eyes is likely to result from suppression of the anti-apoptotic effect of the Ras-pathway at 20-40 h APF by Argos. The loss of pigment cells by overexpressed Argos was also suppressed by the expression of $\operatorname{Ras} 1^{\mathrm{V} 12}$ at the late pupal stage (Figure 4F). It is possible that this effect of Ras $1^{\mathrm{V} 12}$ on pigment cells is caused by both overrecruitment of cells and suppression of PCD.

\section{Dsor1 and rolled function to prevent PCD induced by Hid}

We next examined the function of the Ras pathway in preventing PCD initiated by stimuli other than Argos. To assess the capacity of other downstream components of the Ras pathway to regulate PCD, we analyzed the effects of hyperactivation of MEK and ERK on ectopic cell death caused by the overexpression of Hid in the eyes. Flies carrying one copy of the GMR-hid transgene, where the hid gene was expressed specifically in the developing eyes, exhibited near ablation of the adult compound eyes (Grether et al, 1995; Figure 5A). We found that the GMR-hid phenotype was markedly suppressed by Dsor ${ }^{\mathrm{Su} 1}$ (Tsuda et al, 1993) and $r^{\text {Su23 }}$ (Sawamoto et al, 1996), gain-of-function mutations of the genes encoding MEK and ERK, respectively. The external morphology of the Dsor ${ }^{\text {Su1 } 1}$ eye is indistinguishable from wildtype (Lim et al, 1997), while the $r^{\text {Su23 }}$ flies have roughened but 

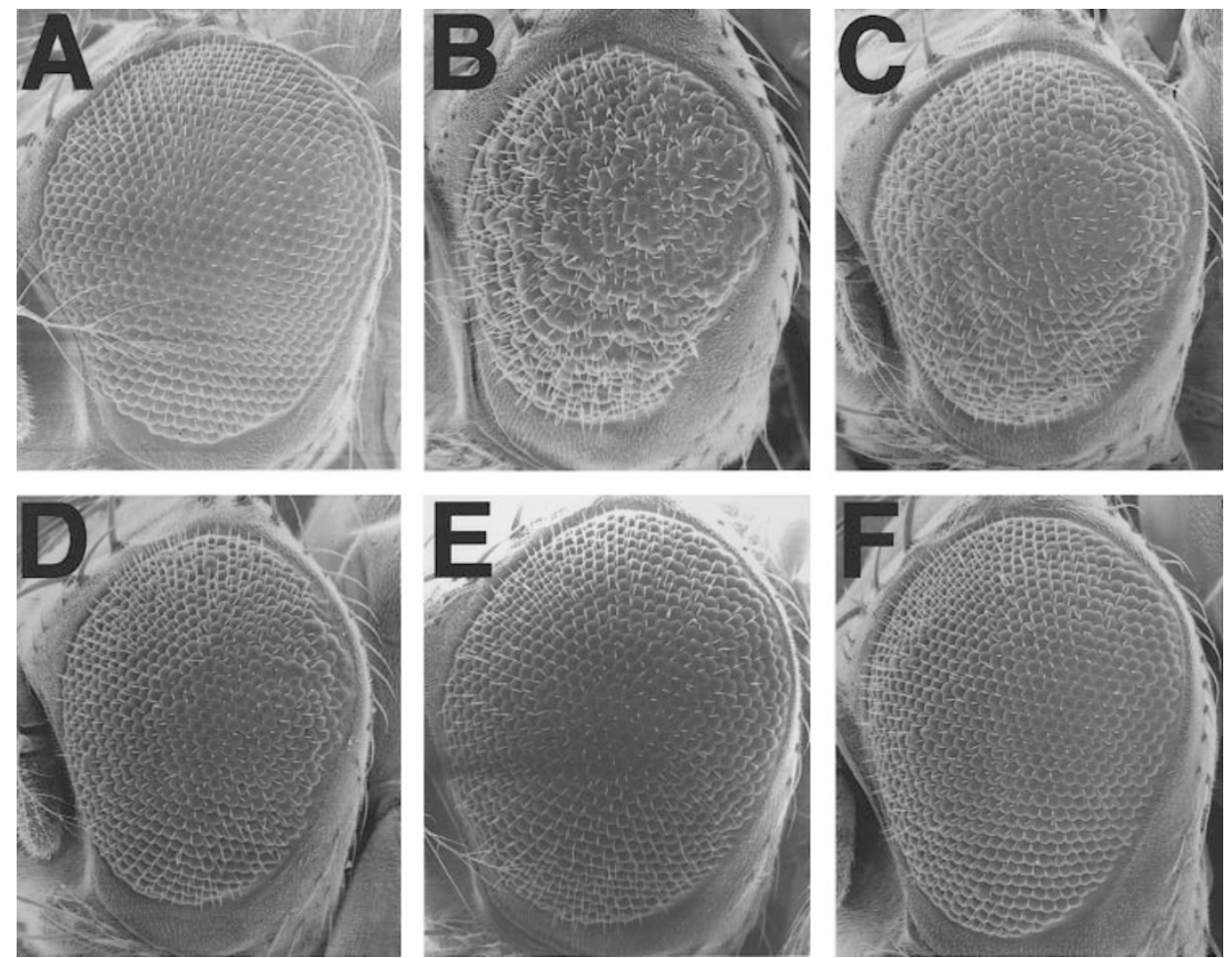

Figure 5 Hyper-activated MEK and MAPK signaling suppress Hid-induced cell death. (A-C) Scanning electron micrographs of compound eyes. (A) GMR-Hid/+ (B) Dsor ${ }^{\text {Su1 }} / Y$; GMR-hid/+. (C) GMR-Hid/r/Su23. Expression of hid in the developing retina causes eye ablation (A). The gain-of-function mutations of the genes encoding for MEK (B) and ERK (C) restore the ommatidia. (D-F) Acridine orange staining of eye discs from GMR-Hid/+(D), Dsor1 1 su1/Y; GMR-hid/+(E), and GMR$\mathrm{Hid}_{/ \mathrm{r}} \mathrm{Su23}^{\mathrm{Su}}(\mathbf{F})$ larvae. Anterior is to the left. Arrows indicate the position of the morphogenetic furrow. High levels of excessive cell death are present posterior to the morphogenetic furrow in the GMR-Hidl+eye discs (D). The excessive cell death induced by Hid was significantly suppressed by the mutations, Dsor ${ }^{\text {Su1 }}$ (E) and $r^{\text {su23 }}(\mathbf{F})$

normal-sized eyes (Sawamoto et al, 1996). The eye tissue removed by Hid was restored in males carrying an $X$ chromosome with the Dsor1 ${ }^{\mathrm{Su} 1}$ mutation (Figure 5B), whereas females heterozygous for the Dsor1 ${ }^{\text {Su1 }}$ mutation showed less suppression (data not shown). The eye-ablation phenotype of GMR-hid was also significantly suppressed by one copy of the $r P^{\mathrm{Su} 23}$ mutation (Figure 5C).

To examine the extent of cell death in larval eye discs, the live discs were labeled with acridine orange. In GMRhid eye discs, increased numbers of dying cells were observed posterior to the morphogenetic furrow (Figure 5D), compared with wild-type (Figure 1A). The Hid-induced cell death in the eye discs was considerably suppressed by either Dsor1 ${ }^{\mathrm{Su} 1}$ (Figure 5E) or $r^{\mathrm{Su} 23}$ (Figure 5F). These results indicate that MEK and ERK act to suppress the ectopic cell death induced by overexpression of Hid.

\section{Discussion}

Ras-signaling in the developing Drosophila eye is triggered by two receptor tyrosine kinases, Sevenless (Sev) and DER. Sev is required for only the R7 photoreceptor cells (reviewed by Zipursky and Rubin, 1994), while DER is used to induce differentiation of all the cell types (Freeman, 1996). Thus, previous studies have proved that the Ras signaling pathway plays a key role in triggering the differentiation of retinal cells (reviewed by Freeman, 1997). In this study, we examined another role for the Ras-signaling system as a negative regulator of $P C D$, using a well-characterized model system, the developing Drosophila eye.

Interfering genetically with the ability of cells to respond to inductive signals that activate the Ras pathway in the larval eye discs leads to cell death (Rebay and Rubin, 1995; Freeman, 1996). Two transgenic lines, GMR-yan ${ }^{A C T}$ (Rebay and Rubin, 1995) and GMR-DN-DER (Freeman, 1996) show impaired neuronal differentiation and excessive cell death in larval eye discs. It is possible that the presence of undifferentiated cells that fail to be recruited due to the blockage of Ras signaling may cause PCD immediately in the larval eye discs, since PCD is caused by various developmental defects independent of the Ras pathway (reviewed by McCall and Steller, 1997). Since Argos also acts as an inhibitor of DER (Schweitzer et al, 1995b) and Ras signaling (Sawamoto et al, 1996), overexpressed Argos could have blocked differentiation by preventing the retinal cells from responding to Spitz, a ligand for DER (Schweitzer et al, 1995). However, the pattern of neuronal differentiation and cell death in the GMR-argos eye discs appeared nearly normal at the third instar. The differences between the eye phenotypes seen in GMR-argos flies at the third instar versus adult flies are likely to have resulted from cell death events occurring in the pupal stage. The idea that enhanced PCD in the pupal eye disc is likely to have caused the reduced number of 
retinal cells in GMR-argos adult flies is supported by the following evidence. First, overexpression of Argos caused excessive cell death in the pupal retina (Freeman, 1994; Figure 1D). Second, the co-expression of the anti-apoptotic gene, diap1, suppressed the eye phenotype of the adult GMR-argos flies (Figure 3; discussed later). Third, there was a reduced number of retinal cells.

The phenotypes caused by one copy of the GMR-argos transgene are different from those of DN-DER and Yan ${ }^{\mathrm{ACT}}$, which are also negative (and possibly stronger) regulators of Ras signaling. DN-DER and $\mathrm{Yan}^{\mathrm{ACT}}$ caused both suppression of cell recruitment and enhanced PCD in third instar eye discs (Rebay and Rubin, 1995; Freeman, 1996). Flies carrying four copies of the GMR-argos transgene showed a small-eye phenotype similar to GMR$D N-D E R$ and GMR-yan ${ }^{A C T}$ (data not shown). Therefore, it is possible that the inhibitory effect of one copy of the GMR-argos transgene on Ras signaling is not strong enough to prevent the onset of cellular differentiation in the larval eye discs, but causes PCD at pupal stages through another mechanism. Thus, the eye of the flies with one copy of the GMR-argos transgene provides us a unique opportunity to investigate the role of Ras signaling in the regulation of PCD during pupal development.

During late pupal development, 1500-2000 cells in the wild-type Drosophila eye are eliminated by PCD to form the ordered cellular lattices of pigment cells (Cagan and Ready, 1989; Wolff and Ready, 1991). Inhibition of this PCD by ectopically expressed anti-apoptotic genes results in the survival of extra secondary and tertiary pigment cells (Hay et al, 1994, 1995). Similar overproduction of the pigment cells can also result from several genetic conditions that activate Ras signaling, e.g., loss-of-function argos mutants (Freeman et al, 1992), overexpression of the secreted Spitz protein (Freeman, 1996), and overexpression of the constitutively-activated Ras1 protein (this paper). On the other hand, the inhibition of DER signaling by the expression of Argos or dominant-negative DER causes a reduction in the number of pigment cells (Sawamoto et al, 1994; Freeman, 1994, 1996). These results suggest the possibility that Ras signaling functions to prevent pigment cell precursors from undergoing normally occurring PCD. Unfortunately, however, the periods for recruitment of the pigment cells by Ras with that for their elimination by PCD overlap, making it difficult to clarify the role of Ras in pigment cell development. Practically, we could not distinguish the effect of the Ras $1^{\mathrm{V} 12}$ expression on the regulation of PCD from its role in differentiation (Figure 4), so we focused on another cell type, the cone cell. It appears that the loss of cone cells in the GMR-argos pupal retina is suppressed by transient expression of the activated Ras 1 protein at 20-40 h APF (Figure 4). During this period, Ras signaling acts to recruit only pigment cells but not cone cells (Figure 4). Therefore, the Argos-induced death of cone cells seems to have resulted from the suppression of an anti-apoptotic function of Ras rather than from impaired cell recruitment. It is also possible that unknown factor(s) produced by pigment cells are responsible for the suppression of cone cell death. It is important to note that the $h s p 70$ promoter is rather leaky. In fact, we observed that the HS-Ras $1^{\mathrm{V} 12}$ transgene could considerably restore the cone cells even without heat shock in the GMR-argos eyes. It is most likely that the restoration of cone cells is due to both of the functions of Ras1, i.e., promotion of cone cell recruitment (caused by the leaky expression) in early pupal development and suppression of cell death at a later stage.

Suppression of the GMR-argos phenotype by coexpression of p35 or the IAP homologues (Figures 2 and 3) indicates that the loss of each cell type in the GMRargos eye is mainly caused by excessive PCD. Since both p35 and the IAPs act as inhibitors of caspases (Bump et al, 1995; Deveralux et al, 1997), inhibition of Ras signaling by Argos may cause PCD through the activation of caspases. Two Drosophila caspases, DCP-1 (Song et al, 1997) and DrICE (Frazer and Evan, 1997) have been identified recently. These two caspases show high homology with mammalian caspases 3 and 6 (CPP32 and $\mathrm{MCH} 2$ ). Both the Drosophila proteins have been shown to cleave p35 as a substrate and induce PCD in cultured cells (Song et al, 1997; Frazer and Evan, 1997), but its function during eye development remains to be elucidated. Further analyses of the phenotypes of the caspase mutants will be required to clarify the role of Ras signaling in the regulation of caspases.

Several candidate genes that act to bridge between Ras signaling and the activation of the caspases may be located at the $75 \mathrm{C} 1-2$ region on the third chromosome, which is known to be essential for the initiation of PCD in Drosophila. Molecular analyses of the genomic region has revealed three genes in the $\mathrm{Df}(3 \mathrm{~L}) \mathrm{H} 99$ interval: rpr (White et al, 1994), hid (Grether et al, 1995), and grim (Chen et al, 1996). All three genes induce PCD when ectopically expressed, and cell death is blocked by co-expression of p35, indicating that these genes induce PCD through the activation of caspases. We found that the $D f(3 L) H 99$ mutation could dominantly suppress the GMR-argos phenotype (Figure 2). Conversely, the eye-ablation phenotype caused by the ectopic expression of hid was suppressed by gain-of-function mutations of the genes coding for MEK and ERK, downstream effectors of Ras (Figure 5). These results suggest that inhibition of the Ras pathway by Argos activates caspases via the activation of genes in the 75C1-2 region including hid. Alternatively, reduced signaling through the Ras pathway may stimulate unknown mechanisms that act in parallel with the genes in the $75 \mathrm{C} 1-2$ region to activate the caspase pathway.

It has been reported that Ras has both pro- and antiapoptotic influences, depending on cell type and applied stimulus in various mammalian cells. For example, NGF promotes cell survival through the Ras/ERK pathway in PC12 cells (Xia et al, 1995). Mice lacking the B-raf protein, a downstream effector of Ras, experience increased apoptosis in their vascular endothelium (Wojnowski et al, 1997). In contrast, Ras induces PCD in fibroblasts through the activation of Raf (Kauffman-Zeh et al, 1997). Thus, it seems likely that the appropriate biological response to Ras activation and its effect on PCD are influenced by both quantitative and qualitative aspects of signal transduction pathways. The present results show that Ras signaling 
functions to inhibit PCD in the developing Drosophila eye. However, it is possible that Ras signaling also has a proapoptotic function in other Drosophila tissues.

Genetic and molecular studies have identified a number of cell-death genes in Drosophila, some of which share homology with known genes composing the evolutionally conserved pathway for PCD (McCall and Steller, 1997). However, other components of the conserved PCD pathway such as ced4 and ced9/bcl-2 have yet to be elucidated. It is possible that these unidentified genes are involved in the regulation of PCD by the Ras pathway. For example, activation of the Ras pathway may suppress PCD through the transcription of ced-9/bcl-2 homologs as reported for mammals (Kinoshita et al, 1995). Genetic screening for modifiers of the GMR-argos phenotype, currently in progress in our laboratory, should identify novel genes involved in the regulation of PCD by the Ras pathway.

\section{Materials and Methods \\ Drosophila stocks and culture}

Flies were raised on a standard cornmeal-glucose-yeast medium at $25^{\circ} \mathrm{C}$, except where otherwise noted. Mutant flies used in this study were GMR-p35 (Hay et al, 1994), GMR-diap1 (Hay et al, 1995), GMRdiap2 (Hay et al, 1995), $r^{\text {Su23 }}$ (Lim et al, 1997), Dsor1 ${ }^{\text {Su1 }}$ (Tsuda et al, 1993), Df(3L)H99 (White et al, 1994), and GMR-hid (Grether et al, 1995). The HS-Ras $1^{\mathrm{V} 12}$ transgenic strain is a gift from N. Perrimon. Canton-S or white ${ }^{1118}$ were used as wild-type strains.

\section{Plasmid construction and P-element mediated germline transformation}

pGMR-argos was constructed by inserting the $2 \mathrm{~kb} E c o R I$ fragment of the argos CDNA including the entire coding region (Okano et al, 1992) into the EcoRI site of the pGMR1 vector (Hay et al, 1994). The resulting pGMR-argos plasmid was injected into $w^{1118}$; Dr/TMS, $S b P\left[r y^{+}, \Delta 2-3\right]$ embryos as previously described (Sawamoto et al, 1994). Four independent transformant lines showing similar rough-eye phenotypes were obtained. All data presented in this paper are from a single strain.

\section{Histology}

Flies were prepared for scanning electron microscopy as described by Kimmel et al (1990). Semi-thin sections of adult heads were prepared as described by Sawamoto et al (1996). Cobalt sulfide staining and acridine orange staining were performed by methods described in Wolff and Ready (1991). Immunohistochemistry of eye discs was carried out essentially as described (Tomlinson and Ready, 1987), except that discs were fixed in $4 \%$ paraformaldehyde in PBS.

\section{Acknowledgements}

We are grateful to Masayuki Miura for critical comments on the manuscript; Kenji Matsuno and Hirotaka Kanuka for discussions; Yasuyoshi Nishida, Norbert Perrimon, Roger Jacobs, Gerald Rubin, and Herman Steller for the fly stocks; Bruce Hay for pGMR; and Developmental Studies Hybridoma Bank for the anti-ELAV antibody. This work was supported by grants from the Ministry of Education, Science, Sports and Culture of Japan to KS and HO.

\section{References}

Bonini NM (1997) Surviving Drosophila eye development. Cell Death Differ. 4: 4-11 Bruuner A, Twardzik T and Schneuly S (1994) The Drosophilagiant lens gene plays a dual role in eye and optic lobe development: Inhibition of differentiation of ommatidial cells and interferes in photoreceptor axon guidance. Mech. Dev. 48: $175-185$

Bump N, Hackett M, Hugunin M, Shagiri S, Brady K, Chen P, Ferenz C, Franklin S, Ghryur T, Li P, Licari P, Mankovich J, Shi L, Greenberg A, Miller L and Wong W (1995) Inhibition of ICE family proteases by baculovirus antiapoptotic protein p35. Science 269: 1885-1888

Cagan RL and Ready DF (1989) The emergence of order in the Drosophila pupal retina. Dev. Biol. 136: 346-362

Chen P, Nordstorm W, Gish B and Abrams J (1996) grim, a novel cell death gene in Drosophila. Genes Dev. 10: 1773-1782

Deveraux QL, Takahashi R, Salvesen GS and Reed JC (1997) X-linked IAP is a direct inhibitor of cell-death proteases. Nature 388: 300-304

Ellis RE, Yuan J and Horvitz HR (1991) Mechanisms and function of cell death. Annu. Rev. Cell Biol. 7: 663-698

Ellis RE and Horvitz HR (1986) Genetic control of programmed cell death in $C$. elegans. Cell 44: 817-829

Fraser AG and Evan GI (1997) Identification of a Drosophila melanogaster ICE/CED3-related protease, drICE. EMBO J. 16: 2805-2813

Freeman M, Klämbt C, Goodman CS and Rubin GM (1992) The argos gene encodes a diffusible factor that regulates cell fate decisions in the Drosophila eye. Cell 69: 963-975

Freeman M (1994) Misexpression of the argos gene, a secreted reglator in cell determination. Development 120: 2297-2304

Freeman M (1996) Reiterative use of the EGF receptor triggers differentiation of all cell types in the Drosophila eye. Cell 87: 651-660

Freeman M (1997) Cell determination strategies in the Drosophila eye. Development 124: $261-270$

Grether ME, Abraham JM, Agapite J, White K and Steller H (1995) The head involution defective gene of Drosophila melanogaster functions in programmed cell death. Genes Dev. 9: 1694-1708

Hay BA, Wolff T and Rubin GM (1994) Expression of baculovirus P35 prevents cell death in Drosophila. Development 120: 2121-2129

Hay BA, Wassarman DA and Rubin GM (1995) Drosophila homologues of baculovirus inhibitor of apoptosis proteins function to block cell death. Cell 83: $1253-1262$

Hengartner MO, Ellis RE and Horvitz HR (1992) Caenorhabditis elegans gene ced-9 protects cells from programmed cell death. Nature 356: 494-499

Hengartner MO and Horvitz HR (1994) The $C$. elegans cell survival gene ced-9 encodes a functional homolog of the mammalian proto-oncogene $b c l-2$. Cell 76 : $665-676$

Kaufmann-Zeh A, Rodriguez-Viciana P, UlrichE, Gilbert C, CofferP, Downward Jand Evan G (1997) Suppression of C-Myc-induced apoptosis by Ras signaling through $\mathrm{PI}(3) \mathrm{K}$ and PKB. Nature 385: 544-548

Kimmel BE, Heberlein U and Rubin GM (1990) The homeo domain protein rough is expressed in a subset of cells in the developing Drosophila eye where it can specify photoreceptor cell subtype. Genes Dev. 4: 712-727

Kinoshita T, Yokota T, Arai K and Miyajima A (1995) Regulation of Bcl-2 expression by oncogenic Ras protein in hematopoietic cells. Oncogene 10: 2207-2212

Kretzschmar D, Brunner A, Wiersdorff V, Pflugfelder GO, Heisenberg M and Schneuwly S (1992) giant lens, a gene involved in cell determination and axonal guidance in the visual system of Drosophila melanogaster. EMBO J. 11: 2531 2539

Lim Y, Tsuda L, Inoue YH, Irie K, Adachi-Yamada T, Hata M, Nishi Y, Matsumoto K and Nishida Y (1997) Dominant mutations of Drosophila MAP kinase kinase and their activities in Drosophila and yeast MAP kinase cascades. Genetics 146: $263-273$

McCall K and Steller H (1997) Facing death in the fly: genetic analysis of apoptosis in Drosophila. Trends Genet. 13: 222-226 
Miura M, Zhu H, Rotello R, Hartwieg EA and Yuan J (1993) Induction of apoptosis in fibroblasts by IL-1 $\beta$-converting enzyme, a mammalian homolog of the $C$. elegans cell death gene ced-3. Cell 75: 653-660

Nordstrom W, Chen P, Steller H and Abrams JM (1996) Activation of the reaper gene during ectopic cell killing in Drosophila. Dev. Biol. 180: 213-226

Okano H, Hayashi S, Tanimura T, Sawamoto K, Yoshikawa S, Watanabe J, Iwasaki M, Hirose S, Mikoshiba K and Montell C (1992) Regulation of Drosophila neural development by a putative secreted protein. Differentiation 52: 1-11

Pritchard C and McMahon M (1997) Raf revealed in life-or-death decisions. Nature Genet. 16: 214-215

Rabizadeh S, LaCount DJ, Friesen PD and Bredesen DE (1993) Expression of the baculovirus p35 gene inhibits mammalian cell death. J. Neurochem. 61:23182321

Rebay I and Rubin GM (1995) Yan functions as a general inhibitor of differentiation and is negatively regulated by activation of the Ras $1 /$ MAPK pathway. Cell 81 : $857-866$

Sawamoto K, Okano H, Kobayakawa Y, Hayashi S, Mikoshiba K and Tanimura T (1994) The function of argos in regulating cell fate decisions during the Drosophila eye and wing vein development. Dev. Biol. 164: 267-276

Sawamoto K and Okano H (1996) Cell-cell interactions during neural development: multiple types of lateral inhibitions involved in Drosophila eye development. Neurosci. Res. 26: 205-214

Sawamoto K, Okabe M, Tanimura T, Mikoshiba K, Nishida Y and Okano H(1996) The Drosophila secreted protein Argos regulates signal transduction in the Ras/MAP kinase pathway. Dev. Biol. 178: 13-22

Schweitzer R, Shaharabany M, SegerR and Shilo BZ(1995a) Secreted Spitz triggers the DER signaling pathway and is a limiting component in embryonic ventral ectoderm determination, Genes Dev. 9: 1518-1529

Schweitzer R, Howes R, Smith R, Shilo BZ and Freeman M (1995b) Inhibition of Drosophila EGF receptor activation by the secreted protein Argos. Nature 376 : $699-702$

Song Z, McCall K and Steller H (1997) DCP-1, a Drosophila cell death protease essential for development. Science 275: 536-540
StellerH(1995) Mechanisms and genes of cellular suicide. Science 267: 1445-1449 Sugimoto A, Friesen PD and Rothman JH (1994) Baculovirus p35 prevents developmentally programmed cell death and rescues a ced-9 mutant in the nematode Caenorhabditis elegans. EMBO J. 13: 2023-2028

Tomlinson A and Ready DF (1987) Neuronal differentiation in the Drosophila ommatidium. Dev. Biol. 123: 264-275

Tsuda L, Inoue YH, Yoo M, Mizuno M, Hata M, Lim Y, Adachi-Yamada T, Ryo H, Masamune $Y$ and Nishida $Y$ (1993) A protein kinase similar to MAP kinase activator acts downstream of the raf kinase in Drosophila. Cell 72: 407-414

Tsujimoto Y, Cossman J, Jaffe E and Croce C (1985) Involvement of the bcl-2gene in human follicular lymphoma. Science 228: 1440-1443

White K, Grether ME, Abrams JM, Young L, Farrell K and Steller H (1994) Genetic control of programmed cell death in Drosophila. Science 264: 677-683

White Kand Steller H (1995) The control of apoptosis in Drosophila. Trends Cell. Biol. 5: $74-78$

White K, Tahaoglu E and Steller H (1996) Cell killing by the Drosophila gene reaper. Science 271: 805-807

Wojnowski L, Zimmer AM, Beck TW, Hahn H, Bernal R, Rapp UR and Zimmer A (1997) Endothelial apoptosis in Braf-deficient mice. Nature Genet. 16: 293-297

Wolff T and Ready DF (1991) Cell death in normal and rough eye mutants of Drosophila. Development 113: 825-839

Xia Z, Dickens M, Raingeaud J, Davis RJ and Greenberg ME (1995) Opposing effects of ERK and JNK-p38 MAPK kinases on apoptosis. Science 270: 1326-1331

Yuan J, Shaham S, Ledoux S, Ellis HM and Horvitz HR (1993) The $C$. elegans cell death gene ced-3 encodes a protein similar to mammalian interleukin- $1 \beta$ converting enzyme. Cell 75: 641-652

Zipursky LR and Rubin GM (1994) Determination of neuronal cell fate: lessons from the R7 neuron of Drosophila. Annu, Rev. Neurosci. 17: 373-397

Zou H, Henzel WJ, Liu X, Lutschg A and Wang Z (1997) Apaf-1, a human protein homologous to $C$. elegans CED-4, participates in cytochrome c-dependent activation of Caspase-3. Cell 90: 405-413 